\title{
Neoplasias quísticas de páncreas: una causa más de dolor abdominal inespecífico. Experiencia terapéutica de 10 años en la Unidad de Cirugía Hepato-Pancreato-Biliar del Hospital General de México
}

\author{
Cystic pancreatic neoplasms: one more cause of nonspecific abdominal pain. \\ Ten-year therapeutic experience in the Hepato-Pancreato-Biliary Surgery Unit \\ of the General Hospital of Mexico
}

Óscar Chapa Azuela, ${ }^{*}$ Clara Verónica Gopar Ochoa, ${ }^{* *}$ Agustín Etchegaray Dondé, ${ }^{* * *}$ Francisco Higuera Hidalgo, $* * *$ Vanessa Ortiz Higareda****

Palabras clave: Páncreas, neoplasias quísticas, carcinoma quístico, dolor abdominal.

Key words: Pancreas, cystic neoplasms, cystic carcinoma, abdominal pain.
* Jefe de Servicio de la Clínica de Páncreas del Hospital General de México (HGM).

** Cirujana General y Endoscopista, Hospital Darío Fernández ISSSTE.

*** Cirujano adscrito al servicio de cirugía HPB, Hospital General de México, SS.

**** Cirujana adscrita al servicio de cirugía general Centro Médico Nacional "Siglo XXI", IMSS.

Recibido: 17/05/2016 Aceptado: 12/07/2017

\section{RESUMEN}

Introducción: Las neoplasias quísticas de páncreas (NQP) se originan a partir de los diversos componentes glandulares; son lesiones infrecuentes que se manifiestan como dolor abdominal crónico e inespecífico. Son subdiagnosticadas. Su conocimiento debe ser difundido con base en que cada estirpe histológica presenta comportamiento biológico distinto y puede progresar a carcinoma invasivo; su diagnóstico y terapéutica en etapas tempranas es fundamental. En el Hospital General de México se llevó a cabo un análisis retrospectivo de los pacientes con NQP tratados en la clínica de patología hepatobiliar. Material y métodos: Se realizó un estudio retrospectivo, descriptivo y transversal de enero de 2004 a diciembre de 2014 en el que se analizaron las características demográficas y clínicas de los pacientes con diagnóstico de NQP que fueron tratados mediante resección quirúrgica; los resultados fueron expresados mediante medidas de tendencia central y porcentajes. Resultados: Se incluyó un total de 18 casos, todos del sexo femenino, con una frecuencia de dos casos por año y una edad promedio de 38 años; la cirugía realizada con mayor frecuencia fue la pancreatectomía distal y en segundo lugar, la pancreatoduodenectomía, con una morbilidad del $39 \%$ y una mortalidad del $6 \%$. Los diagnósticos histopatológicos fueron neoplasia quística mucinosa (NQM) 33\%, neoplasia sólido quística pseudopapilar mucinosa (NSQPM) 33\%, neoplasia quística serosa (NQS) $22 \%$, carcinoma neuroendocrino con degeneración quística (CNQ) 6\%, quiste linfoepitelial (QL) 6\%. La supervivencia global a cinco años fue del 83\%. Discusión: Las NQP constituyen un reto diagnóstico y terapéutico. En nuestro medio, el tratamiento quirúrgico fue curativo en el $83 \%$ de los casos. El abordaje de los pacientes con NQP debe llevarse a cabo en unidades especializadas en patología hepatobiliar, ya que favorece mejores resultados.

\section{ABSTRACT}

Introduction: Pancreatic cystic neoplasms (PCN) originate from various glandular components; these are infrequent lesions that manifest as chronic and non-specific abdominal pain and are under diagnosed. Their knowledge must be diffused on the basis that each histological strain presents different biological behavior and it progresses to invasive carcinoma; its diagnosis and therapeutics in the early stages are critical. A retrospective analysis of patients with PCN treated at the hepatobiliary pathology clinic was carried out at the General Hospital of Mexico. Material and methods: A retrospective, descriptive and cross-sectional study was carried out from January 2004 to December 2014, in which we analyzed the demographic and clinical characteristics of the patients with a diagnosis of PCN, who were treated by surgical resection; the results were expressed through measures of central tendency and percentages. Results: A total of 18 cases, all of them female, with a frequency of two cases per year and a mean age of 38 years. The most frequently performed surgery was distal pancreatectomy and, secondly, pancreatoduodenectomy, with a morbidity of $39 \%$ and a mortality of $6 \%$. Histopathological diagnoses were mucinous cystic neoplasm 33\%, solid cystic neoplasm pseudopapillary mucinous 33\%, serous cystic neoplasm $22 \%$, neuroendocrine carcinoma with cystic degeneration $6 \%$, and lymphoepithelial cyst $6 \%$. Overall survival at five years was $83 \%$. Discussion: PCN is a diagnostic and therapeutic challenge. In our setting, surgical treatment was curative in $83 \%$ of cases. The care of patients with PCN should be carried out in specialized units in hepatobiliary pathology, since it favors better results. 


\section{INTRODUCCIÓN}

$E^{\prime}$ I dolor abdominal ocasiona del 10 al 20\% de los motivos de consulta; ${ }^{1}$ el cirujano general debe distinguir entre sus diversas etiologías para determinar si el tratamiento involucra o no un procedimiento quirúrgico. Dentro de las causas infrecuentes de dolor abdominal crónico se encuentran las neoplasias quísticas de páncreas (NQP).

Las lesiones quísticas de páncreas se dividen en neoplásicas y no neoplásicas; las primeras constituyen el $10 \%$ y se clasifican por estirpes histológicas de acuerdo a su origen a partir de los diversos componentes glandulares, el 90\% restante son quistes inflamatorios, principalmente pseudoquiste de páncreas. El 90\% de las NQP lo conforman cuatro estirpes: la neoplasia intraductal papilar mucinosa con sus variantes de conducto principal, conducto secundario o mixta (NIPM-CP/NIPM-CS/NIPM-M), la neoplasia quística mucinosa (NQM), la neoplasia quística serosa (NQP) y la neoplasia sólido quística pseudopapilar mucinosa (NSQPM); existen otras en menor frecuencia, como las neoplasias neuroendocrinas con degeneración quística y los quistes linfoepiteliales..$^{2-4}$ Su caracterización ha cobrado auge durante los últimos 35 años con el desarrollo de nuevos métodos de imagen que permiten su detección en etapas tempranas y su clasificación basada en elementos morfológicos. La importancia de establecer un diagnóstico diferencial radica en que cada estirpe histológica posee un comportamiento biológico específico, diferente al de las demás, por lo que requerirá un manejo distinto. Las neoplasias de origen mucinoso (NIPM y NQM) presentan progresión hasta el carcinoma invasivo $y$, al igual que los tumores malignos, la etapa clínica al momento de su detección es fundamental en el pronóstico y la supervivencia; existen neoplasias con comportamiento maligno de bajo grado (NSQPM), cuya progresión es lenta y su tratamiento es la resección aún con enfermedad metastásica y neoplasias con comportamiento benigno (NQS), que deben ser tratadas mediante observación, evitando riesgos quirúrgicos innecesarios. ${ }^{5-7}$

Tecnológicamente, se desarrollan métodos que mejoran la precisión en el establecimiento de un diagnóstico diferencial como la biopsia por aspiración guiada por ultrasonido endoscópico (USE) con análisis químico, molecular y citológico de líquido, que mejora los índices de sensibilidad y especificidad mediante el uso concomitante de métodos de imagen no inva$\operatorname{sivos}^{8-11}$ (tomografía computada y resonancia magnética).

El síntoma más frecuente asociado a NQP es el dolor abdominal inespecífico, por lo que fácilmente son confundidas con trastornos gastrointestinales funcionales, lo que retrasa el diagnóstico; el análisis detallado y los estudios de gabinete dan pauta a su sospecha clínica y detección. ${ }^{12}$

Durante los últimos seis años, el estudio y la investigación de las NQP han tenido un gran auge; en el año $2012^{7}$ se llevó a cabo la revisión del consenso internacional sobre el abordaje diagnóstico y terapéutico de las NQP basado en el consenso 2006 y en series de casos internacionales desarrolladas en centros especializados en patología hepatobiliar. A principios de 2016 surgen las guías de la ASGE ${ }^{13}$ (American Society for Gastrointestinal Endoscopy), en las que se menciona el rol que juega la endoscopia en el abordaje diagnóstico y terapéutico de las NQP. Sin embargo, aún existe un gran campo de investigación clínica en el establecimiento del abordaje terapéutico de estas lesiones. Los esfuerzos se enfocan en la determinación de cuáles lesiones resecar y cuáles mantener en vigilancia, tomando en cuenta el grado de progresión maligna, las condiciones físicas del paciente, el riesgo quirúrgico, las secuelas, la morbilidad asociada y la esperanza de vida previa al procedimiento quirúrgico.

El objetivo de este estudio es mostrar la experiencia de 10 años en el tratamiento de las NQP dentro de la Unidad de Cirugía Hepato-Pancreato-Biliar del Hospital General de México.

\section{MATERIAL Y MÉTODOS}

Se realizó un estudio retrospectivo, descriptivo y transversal que incluyó a todos los pacientes con diagnóstico de NQP que fueron llevados a resección quirúrgica de enero de 2004 a diciembre de 2014 en la Unidad de Cirugía Hepato-Pancreato-Biliar del Hospital General de México. Fueron excluidos los casos de NQP 
que no fueron resecados, ya por evidencia de metástasis o por tratarse de lesiones con comportamiento benigno de acuerdo con los criterios internacionales. Se determinaron las variables demográficas de la población estudiada (edad, sexo y características clínicas). Los resultados son expresados en medidas de tendencia central y porcentajes, y la supervivencia con base en el tipo de neoplasia mediante tablas de Kaplan-Meier.

\section{RESULTADOS}

Se incluyó un total de 18 casos, todos de sexo femenino, con una edad promedio de 38 años y una detección promedio de dos casos por año. Las características demográficas de las pacientes estudiadas se muestran en el cuadro $I$.

Todas las pacientes requirieron resección quirúrgica; en 16 casos (83\%), se realizó pancreatectomía distal, 11 (62\%) mediante abordaje abierto y cinco (27\%) laparoscópico; en dos casos (11\%) se realizó pancreatoduodenectomía (cirugía de Whipple clásico) mediante abordaje abierto. El tamaño promedio de las lesiones fue de $10 \pm 4 \mathrm{~cm}$; los diagnósticos histopatológicos encontrados fueron NQM, seis casos (33\%); NSQPM, seis (33\%); NQS, cuatro $(22 \%)$; carcinoma neuroendocrino con degeneración quística $(\mathrm{CNQ})$, uno; (6\%), quiste linfoepitelial (QL), uno (6\%) (Figura 1).

Se identificó displasia leve en dos casos (11\%), correspondientes a neoplasias quísticas mucinosas (borderline), y displasia severa en otros dos (adenocarcinoma mucinoso y adenocarcinoma neuroendocrino con degeneración quística). Uno de los casos de NQM con displasia leve evolucionó durante el seguimiento postoperatorio a carcinoma invasivo, con una supervivencia de 36 meses.

La mortalidad perioperatoria fue del $6 \%$ (un caso, adenocarcinoma mucinoso), asociada a ruptura espontánea de la neoplasia quística, hemoperitoneo y sepsis abdominal. La morbilidad postoperatoria fue del $39 \%$; la complicación más frecuente fue la fístula pancreática, en tres casos (17\%), con resolución espontánea en todos ellos. El tiempo promedio de seguimiento fue de 56 meses y la mortalidad global a cinco años de $17 \%$ (3 casos). La supervivencia con base en el tipo de neoplasia se muestra en la figura 2. La resección quirúrgica fue curativa en 15 casos (83\%), sin evidencia de recidiva a cinco años.

\section{DISCUSIÓN}

En nuestra experiencia, la resección quirúrgica fue curativa en un $83 \%$ de los pacientes trata-

Cuadro I. Características clínicas y demográficas de las pacientes con neoplasias quísticas de páncreas (NQP) tratadas en el Hospital General de México (HGM) 2004-2014.

\begin{tabular}{|c|c|c|c|c|c|c|c|}
\hline Variables & $\begin{array}{c}\text { Todas las NQP } \\
\mathrm{N}=18\end{array}$ & $\begin{array}{l}\text { NIPM } \\
N=0\end{array}$ & $\begin{array}{l}\mathrm{NQM} \\
\mathrm{N}=6\end{array}$ & $\begin{array}{l}\mathrm{NQS} \\
\mathrm{N}=4\end{array}$ & $\begin{array}{l}\text { NSQPM } \\
\mathrm{N}=6\end{array}$ & $\begin{array}{c}\text { Carcinoma } \\
\text { neuroendocrino } \\
\mathrm{N}=1\end{array}$ & $\begin{array}{c}\text { Quiste } \\
\text { linfoepitelial } \\
\mathrm{N}=1\end{array}$ \\
\hline Edad (años) & $38 \pm 13$ & - & $39 \pm 13$ & $49 \pm 17$ & $32 \pm 11$ & 41 & 22 \\
\hline \multicolumn{8}{|l|}{ Sexo } \\
\hline Masculino & 0 & - & 0 & 0 & 0 & 0 & 0 \\
\hline Femenino & $18(100 \%)$ & - & $6(100 \%)$ & $4(100 \%)$ & $6(100 \%)$ & $1(100 \%)$ & $1(100 \%)$ \\
\hline \multicolumn{8}{|l|}{ Presentación clínica } \\
\hline Dolor abdominal & 17 (94\%) & - & $6(100 \%)$ & $4(100 \%)$ & $6(100 \%)$ & 0 & $1(100 \%)$ \\
\hline Ictericia & $1(6 \%)$ & - & 0 & 0 & $1(17 \%)$ & 0 & 0 \\
\hline Pancreatitis & $3(17 \%)$ & - & $1(17 \%)$ & 0 & $1(17 \%)$ & 0 & $1(100 \%)$ \\
\hline Abdomen agudo & $1(6 \%)$ & - & $1(17 \%)$ & 0 & 0 & 0 & 0 \\
\hline $\begin{array}{l}\text { Tiempo de evolución previo al } \\
\text { diagnóstico (meses) }\end{array}$ & $8 \pm 9$ & - & $9 \pm 13$ & $7 \pm 4$ & $5 \pm 4$ & 2 & 24 \\
\hline
\end{tabular}




\begin{tabular}{|c|c|c|c|c|c|c|c|}
\hline \multicolumn{8}{|c|}{ Continúa cuadro I... } \\
\hline Variables & $\begin{array}{c}\text { Todas las NQP } \\
\mathrm{N}=18\end{array}$ & $\begin{array}{l}\text { NIPM } \\
\mathrm{N}=0\end{array}$ & $\begin{array}{l}\mathrm{NQM} \\
\mathrm{N}=6\end{array}$ & $\begin{array}{l}\mathrm{NQS} \\
\mathrm{N}=4\end{array}$ & $\begin{array}{l}\text { NSQPM } \\
\mathrm{N}=6\end{array}$ & $\begin{array}{c}\text { Carcinoma } \\
\text { neuroendocrino } \\
\mathrm{N}=1\end{array}$ & $\begin{array}{c}\text { Quiste } \\
\text { linfoepitelial } \\
\mathrm{N}=1\end{array}$ \\
\hline \multicolumn{8}{|l|}{ Estudios de imagen } \\
\hline $\mathrm{TAC}$ & $18(100 \%)$ & - & $6(100 \%)$ & $4(100 \%)$ & $6(100 \%)$ & $1(100 \%)$ & $1(100 \%)$ \\
\hline $\mathrm{RM} / \mathrm{CRM}$ & - & - & - & - & - & - & - \\
\hline CPRE & - & - & - & - & - & - & - \\
\hline USE & - & - & - & - & - & - & - \\
\hline USE + FNA & - & - & - & - & - & - & - \\
\hline \multicolumn{8}{|l|}{ Localización de la lesión } \\
\hline Cabeza & $2(11 \%)$ & - & 0 & 0 & $1(17 \%)$ & $1(100 \%)$ & 0 \\
\hline Cuerpo & $10(56 \%)$ & - & $3(50 \%)$ & $2(50 \%)$ & $4(66 \%)$ & 0 & $1(100 \%)$ \\
\hline Cola & $5(28 \%)$ & - & $2(33 \%)$ & $2(50 \%)$ & $1(17 \%)$ & 0 & 0 \\
\hline $\begin{array}{l}\text { Involucro de todo } \\
\text { el páncreas }\end{array}$ & $1(6 \%)$ & - & $1(17 \%)$ & 0 & 0 & 0 & 0 \\
\hline \multicolumn{8}{|l|}{ Tipo de resección quirúrgica } \\
\hline Cirugía de Whipple & $2(11 \%)$ & - & 0 & 0 & $1(17 \%)$ & $1(100 \%)$ & 0 \\
\hline Pancreatectomía distal & $16(89 \%)$ & - & $6(100 \%)$ & $4(100 \%)$ & $5(83 \%)$ & 0 & $1(100 \%)$ \\
\hline Pancreatectomía media & 0 & - & 0 & 0 & 0 & 0 & 0 \\
\hline Pancreatectomía total & 0 & - & 0 & 0 & 0 & 0 & 0 \\
\hline Tamaño del quiste (cm) & $10 \pm 4$ & - & 13 & 7 & 8 & 9 & 15 \\
\hline $\begin{array}{l}\text { Resultado } \\
\text { histopatológico }\end{array}$ & - & - & $\begin{array}{c}\text { NQM sin } \\
\text { displasia } \\
\text { NQM con } \\
\text { displasia leve, } \\
\text { dos casos } \\
\text { NQM con } \\
\text { displasia severa, } \\
\text { un caso }\end{array}$ & $\begin{array}{l}\text { NQS cuatro } \\
\text { casos/uno con } \\
\text { variedad } \\
\text { poligoquístico }\end{array}$ & $\begin{array}{l}\text { NSQPM seis } \\
\text { casos }\end{array}$ & $\begin{array}{c}\text { Carcinoma } \\
\text { neuroendocrino } \\
\text { con degeneración } \\
\text { quística }\end{array}$ & $\begin{array}{c}\text { Quiste } \\
\text { linfoepitelial }\end{array}$ \\
\hline \multicolumn{8}{|l|}{$\begin{array}{l}\text { Grados de displasia en la } \\
\text { pieza quirúrgica }\end{array}$} \\
\hline Sin displasia & $14(78 \%)$ & - & $3(16 \%)$ & $4(22 \%)$ & $6(34 \%)$ & 0 & $1(6 \%)$ \\
\hline Displasia leve & $2(11 \%)$ & - & $2(11 \%)$ & 0 & 0 & 0 & 0 \\
\hline Displasia severa & $2(11 \%)$ & - & $1(6 \%)$ & 0 & 0 & $1(6 \%)$ & 0 \\
\hline \multirow[t]{3}{*}{ Complicaciones postquirúrgicas } & $7(33 \%)$ & & $\begin{array}{c}3(16.5 \%) \\
\text { Infección de } \\
\text { sitio quirúrgico } \\
\text { superficial }\end{array}$ & $\begin{array}{c}1(6 \%) \\
\text { Fístula } \\
\text { pancreática }\end{array}$ & $\begin{array}{c}2(16.5 \%) \\
\text { Hernia } \\
\text { postincisional }\end{array}$ & $\begin{array}{c}1(6 \%) \\
\text { Fístula } \\
\text { pancreática }\end{array}$ & $\begin{array}{l}- \\
-\end{array}$ \\
\hline & & & $\begin{array}{c}\text { Hernia } \\
\text { postincisional }\end{array}$ & - & $\begin{array}{c}\text { Fístula } \\
\text { pancreática }\end{array}$ & - & - \\
\hline & & & Sepsis & - & - & - & - \\
\hline Mortalidad perioperatoria & $1(6 \%)$ & & $1(17 \%)$ & 0 & 0 & 0 & 0 \\
\hline $\begin{array}{l}\text { Tiempo de seguimiento } \\
\text { promedio (meses) }\end{array}$ & 45 & & 56 & 60 & 55 & 6 & 49 \\
\hline $\begin{array}{l}\text { Progresión maligna } \\
\text { durante el seguimiento }\end{array}$ & $1(5.5 \%)$ & & $1(5.5 \%)$ & 0 & 0 & 0 & 0 \\
\hline Supervivencia global a 5 años & $15(83 \%)$ & & $4(67 \%)$ & $4(100 \%)$ & $6(100 \%)$ & - & $1(100 \%)$ \\
\hline
\end{tabular}


dos; el riesgo de recidiva o progresión de la enfermedad a carcinoma estuvo relacionado con la estirpe histológica de la lesión, lo que concuerda con la experiencia de otros centros

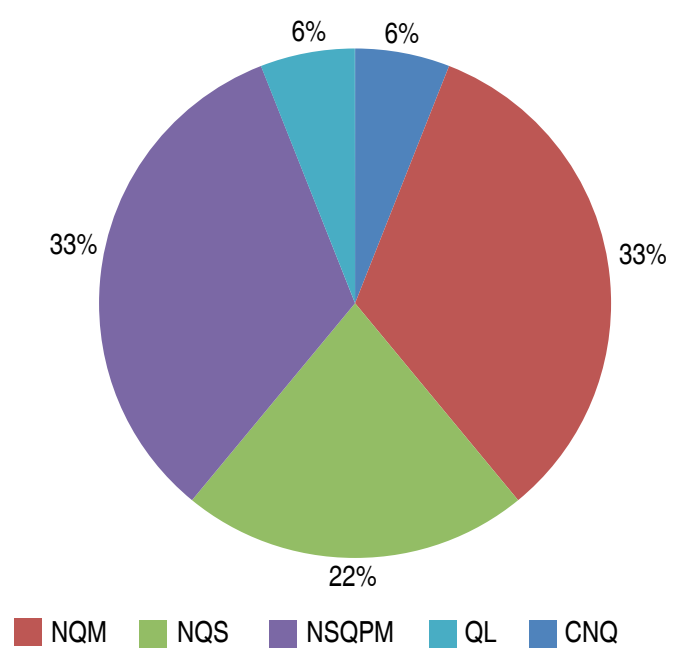

Figura 1. Diagnósticos histopatológicos encontrados en los pacientes con NQP en el HGM de 2004 a 2014.

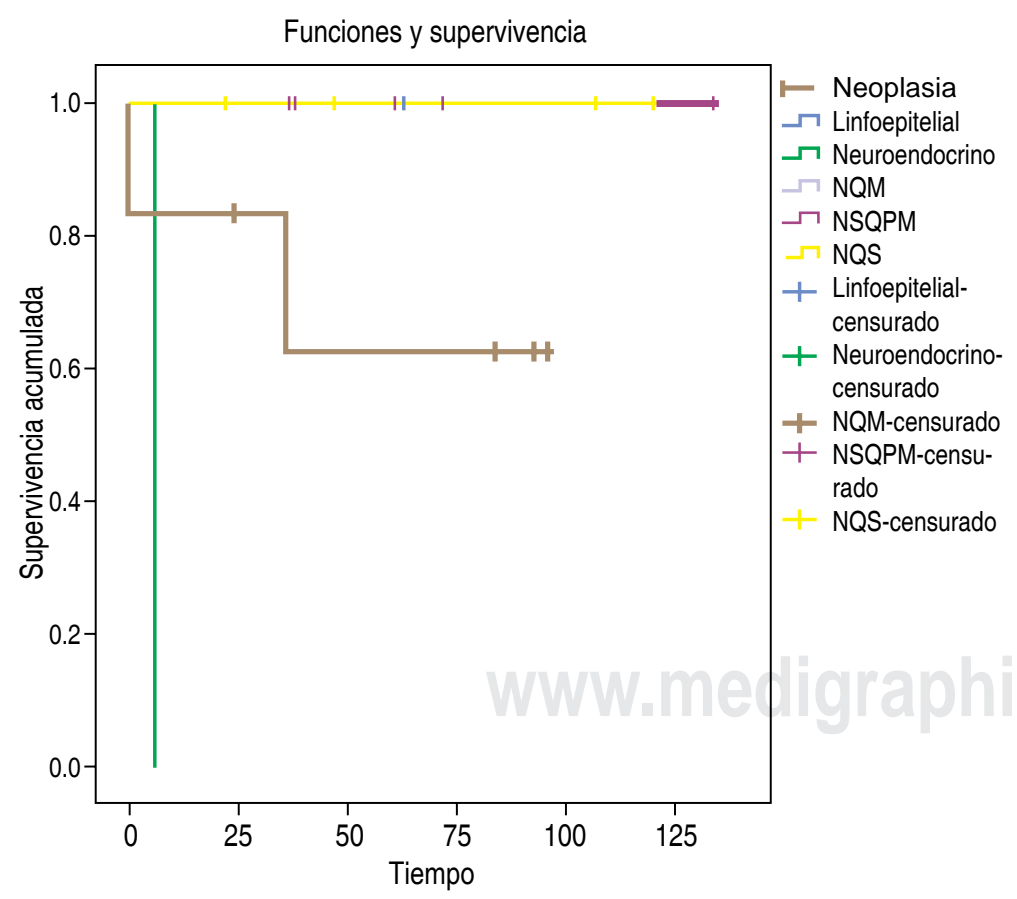

Figura 2. Tablas de supervivencia de pacientes con NQP tratados mediante resección quirúrgica en el HGM 2004-2014. internacionales especializados en patología pancreatobiliar, en los que se ha identificado que es del $17 \%$ para NQM y del $67 \%$ para la NIPM a cinco años. ${ }^{5}$ La morbilidad asociada con la resección quirúrgica para el tratamiento de las NQP, de acuerdo con la serie internacional con mayor número de pacientes, ${ }^{14}$ se sitúa entre el $36.4 \%$ para pancreatectomía distal y el $40 \%$ para pancreatoduodenectomía; en nuestro estudio la morbilidad global fue del $39 \%$ y se relacionó con la complejidad del procedimiento quirúrgico, por lo que se recomienda que el tratamiento de este tipo de neoplasias sea llevado a cabo en unidades de cirugía hepatobiliar como la de nuestro hospital, lo que favorece un manejo multidisciplinario con buenos resultados. Así mismo, el abordaje laparoscópico para patología pancreática mejora los tiempos de estancia intrahospitalaria y la reintegración de los pacientes a sus actividades socioeconómicas; ${ }^{15}$ en todos los casos tratados mediante abordaje laparoscópico, se obtuvieron resultados satisfactorios.

En cuanto a la mortalidad perioperatoria, ocurrió en un caso relacionada a sepsis abdominal por ruptura espontánea de un cistadenocarcinoma mucinoso, lo cual únicamente había sido reportado en otro tipo de neoplasias quísticas como el cistadenocarcinoma seroso, ${ }^{16}$ pero no en lesiones mucinosas; el tratamiento quirúrgico de urgencia y el deterioro de las condiciones de la paciente contribuyeron con el desenlace.

En uno de los casos reportados con displasia leve, hubo progresión de la enfermedad a carcinoma metastásico, lo cual concuerda con lo reportado en la literatura en cuanto a que en una lesión pueden coexistir focos de displasia leve y severa, por lo que el análisis histopatológico debe ser minucioso en este tipo de lesiones. ${ }^{3,5-7}$ Destaca, además, que a pesar de corroborarse la existencia de carcinoma invasivo, las NQM y las NIPM cursan con menor agresividad que el adenocarcinoma ductal de páncreas, este último con una supervivencia de seis a 12 meses, mientras que para las primeras, la supervivencia promedio es de 36 a 60 meses. ${ }^{5,7}$

Las neoplasias quísticas de páncreas continúan siendo un reto diagnóstico y terapéutico; en nuestro estudio se mantuvo un diagnóstico promedio de dos casos por año, lo que nos 
instiga a difundir la información acerca de sus características para favorecer su detección y referencia a centros especializados.

A partir del consenso internacional de 2012, se han modificado los estándares de manejo. Se permite la utilización de recursos diagnósticos como el USE y se amplían los criterios en la toma de decisiones con base en la caracterización de lesiones a partir de elementos morfológicos en nuestro hospital como punto de partida para la selección de pacientes candidatos a resección. Por otro lado, aún existen puntos inconclusos en los que no se ha llegado a un consenso, como el manejo de las neoplasias intraductales de rama secundaria y el uso de quimioterapia intraquística instilada mediante USE, ${ }^{17,18}$ por lo que el estudio y terapéutica de estas lesiones continúa en investigación y modificación constante, y es importante conocerlas para favorecer el pronóstico de nuestros pacientes.

\section{REFERENCIAS}

1. Rivera-Hernández ME, Aguirre-Gas H, RosalesDelgado F, Blanco-Benavidez R. Recomendaciones específicas para el manejo del síndrome doloroso abdominal en los servicios de urgencias. Rev CONAMED. 2007; 12: 4-23.

2. International Agency for Research on Cancer (IARC). WHO classification of tumors. Cap 10: Tumors of the exocrine pancreas. 2000. pp. 219-250.

3. Klimstra D, Kloppel G. Tumors of the exocrine pancreas. Diagnostic histopathology of tumors. 4th ed. Saunders/Elsevier; 2013. pp. 531-558.

4. Assifi MM, Nguyen PD, Agrawal N, Dedania N, Kennedy EP, Sauter PK, et al. Non-neoplastic epithelial cysts of the pancreas: a rare, benign entity. J Gastrointest Surg. 2014; 18: 523-531.

5. Farrell JJ, Fernández-del Castillo C. Pancreatic cystic neoplasms: management and unanswered questions. Gastroenterology. 2013; 144: 1303-1315.

6. Gardner TB, Glass LM, Smith KD, Ripple GH, Barth RJ, Klibansky DA, et al. Pancreatic cyst prevalence and the risk of mucin-producing adenocarcinoma in US adults. Am J Gastroenterol. 2013; 108: 1546-1550.

7. Tanaka M, Fernández-del Castillo C, Adsay V, Chari S, Falconi M, Jang JY, et al. International consensus guidelines 2012 for the management of IPMN and MCN of the pancreas. Pancreatology. 2012; 12: 183197.

8. Sainani NI, Saokar A, Deshpande V, Fernández-del Castillo C, Hahn P, Sahani DV. Comparative performance of MDCT and MRI with MR cholangiopancreatography in characterizing small pancreatic cysts. AJR Am J Roentgenol. 2009; 193: 722-731.

9. Kadiyala V, Lee LS. Endosonography in the diagnosis and management of pancreatic cysts. World J Gastrointest Endosc. 2015; 7: 213-223.

10. Matthaei H, Feldmann G, Lingohr P, Kalff JC. Molecular diagnostics of pancreatic cysts. Langenbecks Arch Surg. 2013; 398: 1021-1027.

11. Ngamruengphong S, Bartel MJ, Raimondo M. Cyst carcinoembryonic antigen in differentiating pancreatic cysts: a meta-analysis. Dig Liver Dis. 2013; 45: 920926.

12. Drossman DA. Rome III: the new criteria. Chin J Dig Dis. 2006; 7: 181-185.

13. ASGE Standards of Practice Committee, Muthusamy VR, Chandrasekhara V, Acosta RD, Bruining DH, Chathadi KV, et al. The role of endoscopy in the diagnosis and treatment of cystic pancreatic neoplasms. Gastrointest Endosc. 2016; 84: 1-9.

14. Valsangkar NP, Morales-Oyarvide V, Thayer SP, Ferrone CR, Wargo JA, Warshaw AL, et al. 851 resected cystic tumors of the pancreas: a 33-year experience at the Massachusetts General Hospital. Surgery. 2012; 152: S4-12.

15. Al-Taan OS, Stephenson JA, Briggs C, Pollard C, Metcalfe MS, Dennison AR. Laparoscopic pancreatic surgery: a review of present results and future prospects. HPB (Oxford). 2010; 12: 239-243.

16. Bramis K, Petrou A, Papalambros A, Manzelli A, Mantonakis E, Brennan N, et al. Serous cystadenocarcinoma of the pancreas: report of a case and management reflections. World J Surg Oncol. 2012; 10: 51.

17. Sahora K, Mino-Kenudson M, Brugge W, Thayer SP, Ferrone CR, Sahani D, et al. Branch duct intraductal papillary mucinous neoplasms: does cyst size change the tip of the scale? A critical analysis of the revised international consensus guidelines in a large singleinstitutional series. Ann Surg. 2013; 258: 466-475.

18. Crippa S, Partelli S, Tamburrino D, Falconi M. The natural history of a branch-duct intraductal papillary mucinous neoplasm of the pancreas. Surgery. 2014; 155: 578-579.

Correspondencia:

Clara Verónica Gopar Ochoa

E-mail: clínicadepancreas@gmail.com cveronicahg@gmail.com 